\title{
Design of the monitoring system for the internal state characteristic parameters of ultra-high voltage metal oxide arresters
}

\author{
Lina Geng ${ }^{1 *}$, Yiling $\mathrm{Ma}^{1}$, Xuchen $\mathrm{Lu}^{1}$ and Yanan Wang ${ }^{1}$ \\ ${ }^{1}$ State Grid Liaoning Electric Power Co., Ltd. Electric Power Research Institute, 110006, Shenyang Liaoning, China
}

\begin{abstract}
At present, UHV surge arresters have a large number of sections and complex structures. The existing live detection and online monitoring technologies can no longer effectively determine the problem of deterioration of the internal resistance of the arrester. This paper studies the structure, power supply, temperature and humidity measurement and wireless transmission methods of measuring probes that can be placed in the arrester for a long time. By measuring the temperature, humidity and current data of each node, and sending it to the background wirelessly, it is more effective to judge the UHV Internal defects of the arrester. The method of internally measuring the temperature and humidity of the arrester node can improve the diagnostic ability of the internal defect and defect location of the UHV arrester, and realize the effective identification and location of the internal fault of the UHV arrester.
\end{abstract}

\section{Introduction}

Preliminary research has found that: After the internal insulating arrester appears on the upper part of the UHV surge arrester, such as the internal resistance piece is damp or aging, the change of the full current and resistive current at the bottom is not obvious. The existing live detection and online monitoring methods are not sensitive enough to judge the internal defects and realize fault location Accurately. Through experimental research, the deterioration process of the arrester can be effectively judged by the local heating of the arrester resistor, and the damp defect that often occurs in the arrester can be sensed by the measurement of internal temperature and humidity. Therefore, only by measuring the temperature and humidity changes inside each section of the arrester can the internal defects of the arrester be found effectively and accurately, and the specific fault section can be located. This paper studies the internal temperature and humidity monitoring system of metal oxide arresters with voltage levels of $500 \mathrm{kV}$ and above. The designed measuring probe is placed in series on the upper part of each section of the arrester, and electricity is obtained through the leakage current of the arrester, combined with the low-power operation mode, and the external host of the arrester. It communicates with the probe wirelessly, transmits the internal temperature and humidity data remotely, and finally displays it in the background interface to achieve the purpose of accurately and effectively discovering the operation defects of the arrester, providing basic data for the operation of the arrester and guiding the UHV Formulation of the maintenance plan for the arrester condition ${ }^{[1-5]}$.

\section{Design of Power Acquisition Module}

The measuring probe designed in this paper is placed inside the arrester for a long time, it can withstand the impact of overvoltage, and under the normal operation of the arrester, it can use the internal leakage current to supply power by itself. The power supply method adopts the mutual inductor induction method, which can sense leakage currents greater than $0.5 \mathrm{~mA}$, and the secondary current is output to TI company's BQ25570 micro-energy harvesting circuit. The circuit is shown in Figure 1.

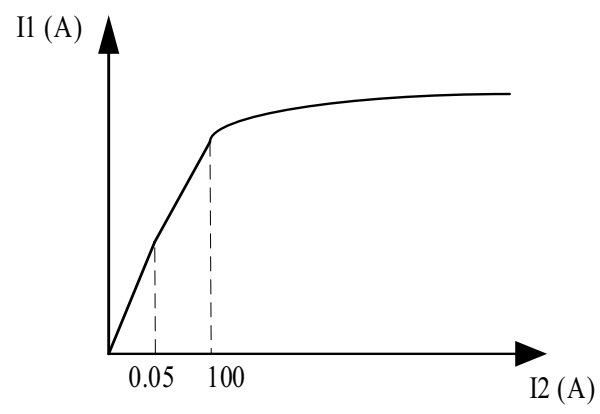

Figure 1. Transformer saturation curve

This paper takes $1000 \mathrm{kV}$ UHV AC lightning arrester as an example. Under normal operating voltage, the leakage current inside the arrester is about $15 \mathrm{~mA}$. When there is a lightning strike or operating overvoltage, the current value will suddenly increase to several hundred kiloamps. To ensure the measurement accuracy of the arrester's leakage current and resistive current, it is also necessary to consider the impact of the large current

\footnotetext{
"Corresponding e-mail: d17640276822@163.com
} 
released by the arrester on the monitoring device. According to the standard of JB/T10492-2011 "Monitor for Metal Oxide Arrester": 1000kV arrester monitor needs to operate normally after $2000 \mathrm{uS}$ square wave (peak) $8000 \mathrm{~A}$ has passed, and the sampling method of resistors is difficult to meet the standard requirements. This paper proposes to use the sampling method of the transformer to solve the above-mentioned problems, and a bipolar transformer is designed that can induce energy in a small current.

TI's BQ25570 micro-energy harvesting program can collect the continuous secondary small energy of the transformer into the super capacitor for storage. When the capacitor is fully charged, the host sends a data command, and the energy of the super capacitor is collected at the back end. The circuit performs a collection and data transmission process. As shown in Figure 2.

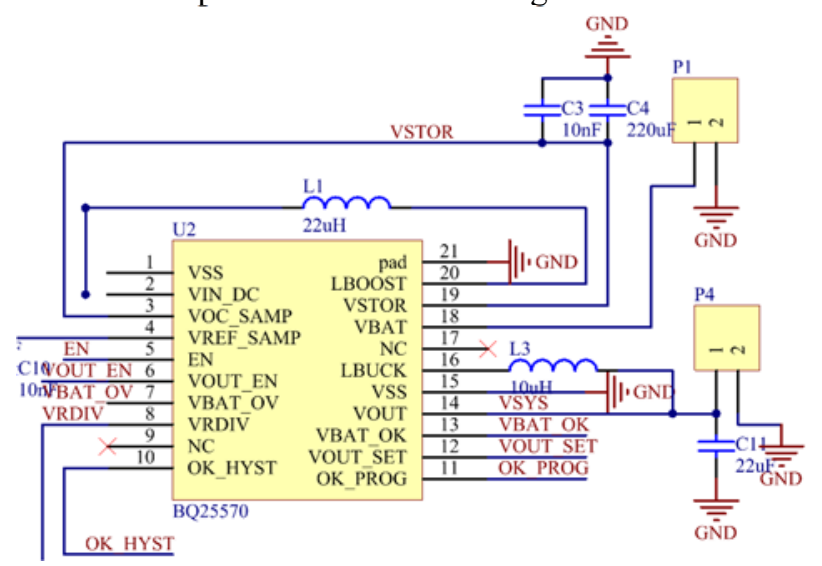

Figure 2. Micro energy harvesting circuit

\section{Design of temperature and humidity acquisition module}

The temperature and humidity sensor adopts the SHT35 temperature and humidity sensor chip from Sensirion, Switzerland, and communicates with the MCU in the circuit through UART. It uses Sensirion's CMOSens technology, highly integrated capacitive humidity sensor and energy gap temperature sensor, and has the advantages of low power consumption, fast response and strong antiinterference ability. This paper uses the BT3110 electronic switch, which can disconnect the temperature and humidity chip when it does not need to work. When the probe receives the acquisition command, the electronic switch is turned on to supply power to the acquisition circuit to achieve temperature and humidity acquisition and measurement, so as to cooperate with the energy module. The working logic can greatly reduce the power consumption of the entire circuit [6-8]. As shown in Figure 3.
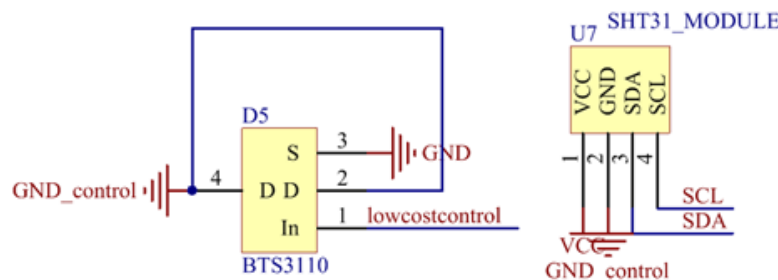

Figure 3. Temperature and humidity chip control circuit diagram

\section{Design of Leakage Current Acquisition System}

Due to the nonlinear characteristics of the arrester, its leakage current is a non-sine wave, which contains highorder harmonics. The fundamental wave of the arrester leakage current can be measured by the harmonic analysis method. The advantage is that the software algorithm filters out the higher harmonics, and more accurately obtains the current parameters that affect the life of the arrester, and improves the accuracy of judging the operating conditions of the MOA. The software calculation formula is as follows:

Considering that the signal contains high-order harmonics and noise, the signal $\mathrm{X}(\mathrm{t})$ can be expressed as:

$$
X(t)=\sum_{k=0}^{+\infty} X_{k} \sin \left(n \omega t+\varphi_{k}\right)+N(t)
$$

According to the orthogonality of trigonometric functions, we have:

$$
\begin{aligned}
& A_{k}=\frac{2}{T} \int_{0}^{T} X(t) \sin \omega t d t=X_{k} \cos \varphi_{k} \\
& B_{k}=\frac{2}{T} \int_{0}^{T} X(t) \cos \omega t d t=X_{k} \sin \varphi_{k}
\end{aligned}
$$

The above is for the time continuous signal. For the discrete signal after sampling, the integral can be expressed as a discrete form:

$$
\begin{aligned}
& A_{k}=\frac{2}{N} \sum_{n=0}^{N-1} X(n) \sin \left(\frac{2 \pi}{N} k n\right) \\
& B_{k}=\frac{2}{N} \sum_{n=0}^{N-1} X(n) \cos \left(\frac{2 \pi}{N} k n\right)
\end{aligned}
$$

$\mathrm{N}$ is the number of sampling points in a period.

Considering that the higher harmonic content in the system is small, the even harmonic content is extremely small. Through the above formula, the DFT transform only decomposes the odd harmonics, and the hardware circuit in the frequency domain has filtered out the odd harmonics greater than 7 th. From this, the amplitude of the fundamental current can be calculated. After such processing, the calculation method conforms to the IEC60099-5 standard, which ensures the accuracy of the lightning arrester leakage current extraction algorithm.

The device accuracy test method is to use a standard source to give a standard signal, compare the measured data of the device and calculate the error. For example, the standard source outputs a total current of $0.49 \mathrm{~mA}$ and a third harmonic current of $0.01 \mathrm{~mA}$. The device collects 256 points per cycle. After the full waveform data is output, 
the device displays a total current of $0.48 \mathrm{~mA}$ and a resistive current of $0.01 \mathrm{~mA}$.

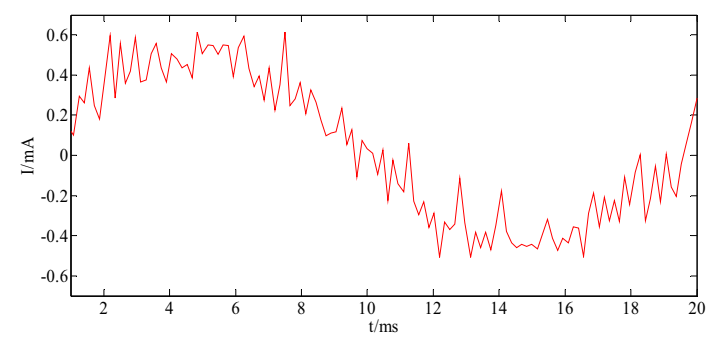

Figure 4. The waveform of acquire current data

According to the measurement range of the device, the accuracy experiment is carried out. The measurement error of the device for the leakage current and resistive current of the arrester is $\leq 1 \%$, which fully meets the measurement accuracy required by the Q/GDW 537-2010 specification.

\section{The design of wireless transmission system}

This paper uses LoRaWAN, which is a highly integrated low-power wireless control module, mainly using LoRa remote modulation and demodulation technology, built-in STM32L series CPU, used for ultra-long-distance spread spectrum communication, which minimizes current consumption while greatly improving Improve the antiinterference and sensitivity. LWM supports three protocol modes of LoRaWAN, which is Class A, B, C. LoRa supports the high-performance (G)FSK mode of WM-Bus and IEEE802.15.4g systems. LWM uses RF solutions to significantly reduce current consumption and significantly optimizes phase noise, selectivity, receiver linearity, thirdorder input intercept point (IIP3) and other performance.

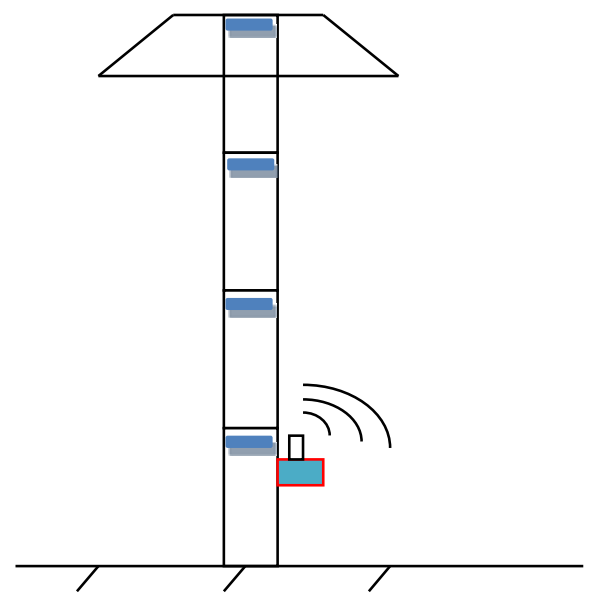

Figure 5. Schematic diagram of communication between measuring probe and host

The developed host is installed on the outside of the arrester, and is powered by an external wired 220VAC. The probes are distributed on the upper part of the arrester, which is convenient for measuring the temperature and humidity of the arrester, as shown in Figure 4. Each probe node composes a LoRaWAN network and is in sleep state most of the time. When the host sends a wake-up command, the probe works, and the measurement data is sent to the host. After the host obtains the data of each probe node, it is transmitted to the host after analysis and calculation. The communication method can be RS485 wired communication or wireless communication [9-10].

\section{Standardized design of measurement data}

Ultra-high voltage arrester metal oxide arrester internal temperature, humidity and current measurement device, the measurement data is sent to the host device through the LORA wireless method, and the data measurement is the response method of the host, which conforms to the MODBUS-RTU protocol. Because the smart substation requires data standardization and conforms to the IEC61850 protocol, the host uses wireless means to interact with the background. This paper studies the standardized access interface and specifications of substation equipment, which is applied to the monitoring system of the internal characteristic parameters of metal oxide arresters of ultra-high voltage arresters. The backend server and the on-site host realize communication standard interface connection, model access, data query, alarm, call, periodic upload, replacement, setting and control (optional) and other communication functions. As shown in Figure 6.

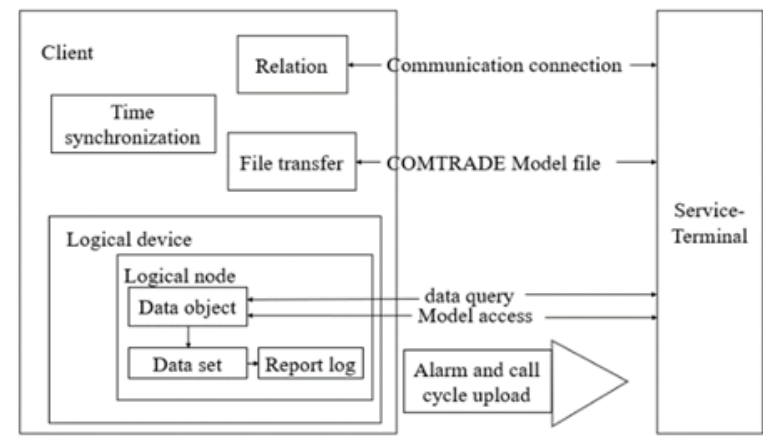

Figure 6. Data standardization interaction block diagram between client and server

The main functions of the standardized interface include monitoring the communication interaction process between the system host and the remote host (CAC), and the service methods provided by both parties. Including the seven processes of heartbeat detection, data upload, data call, data retransmission, read configuration, write configuration and remote update. The wireless transmission adopts DIES encryption technology to ensure the safety and reliability of information transmission. The back-end integrated management system adopts hierarchical authority management to ensure access security.

\section{Conclusion}

This paper studies the temperature, humidity and current measurement methods of each node in the arrester and the 
fusion technology of wireless transmission, which is based on wireless sensor network technology, combined with micro-current power technology. This method can operate the overvoltage measuring probe, adopt the self-pickup method to realize reliable power supply, and transmit the measurement result to the external terminal through the wireless sensor network through the lightning arrester jacket. This can effectively improve the ability to diagnose the internal defects and defect locations of the UHV arrester, realize the effective identification and location of the internal fault of the UHV arrester, and improve the safe operation level of the UHV arrester.

\section{References}

1. J.H. Ma, China Metal Bulletin, 11, 294-295 (2018).

2. H. Han, X.P. Pan, Journal of Hohai University (NATURAL SCIENCE EDITION), 45(3), 277-282 (2017).

3. H.L. Cao, Nanjing University of information technology (2016).

4. X.G. Gao, University of Electronic Science and technology (2018).

5. H.X. Wang, H.F. Liu, et al, Hebei electric power technology, 31(6), 20-22 (2012).

6. K.H. Fu, X.N. Zhang, et al, Agricultural science and technology and equipment, 5, 29-30, 33 (2017).

7. Z.H. Zhang, Z.Y. Wang, et al, Modern electronic technology, 17,137-139 (2009).

8. S.T. Zheng, Electronic production, 12,92-93 (2019).

9. M. Zhu, C.L. Yang, et al, Acta Instrumenta Sinica,34(01), 200-207 (2013).

10. B.P. Tang, Q.Q. Huang, et al, Vibration, test and diagnosis, 34(1), 1-7 (2014). 